\title{
Absorption Enhancement in Peridinin-Chlorophyll-Protein Light-Harvesting Complexes Coupled to Semicontinuous Silver Film
}

\author{
Nikodem Czechowski • Piotr Nyga • \\ Mikołaj K. Schmidt • Tatas H. P. Brotosudarmo • \\ Hugo Scheer • Dawid Piatkowski • Sebastian Mackowski
}

Received: 5 July 2011 / Accepted: 17 August 2011 /Published online: 24 August 2011

(C) The Author(s) 2011. This article is published with open access at Springerlink.com

\begin{abstract}
We report on experimental and theoretical studies of plasmon-induced effects in a hybrid nanostructure composed of light-harvesting complexes and metallic nanoparticles in the form of semicontinuous silver film. The results of continuous-wave and time-resolved spectroscopy indicate that absorption of the light-harvesting complexes is strongly enhanced upon coupling with the metallic film spaced by $25 \mathrm{~nm}$ of a dielectric silica layer. This conclusion is corroborated by modeling, which confirms the morphology of the silver island film.
\end{abstract}

Keywords Light-harvesting complex . Chlorophyll . Absorption enhancement Plasmon excitation .

Fluorescence $\cdot$ Time-resolved fluorescence $\cdot$ Artificial light harvesting $\cdot$ Hybrid nanostructure

\footnotetext{
N. Czechowski $\cdot$ M. K. Schmidt • D. Piatkowski

S. Mackowski $(\square)$

Institute of Physics, Nicolaus Copernicus University,

Grudziadzka 5/7,

87-100 Torun, Poland

e-mail: mackowski@fizyka.umk.pl

\section{P. Nyga}

Institute of Optoelectronics, Military University of Technology,

Gen. Sylwestra Kaliskiego 2,

00-908 Warsaw, Poland
}

T. H. P. Brotosudarmo

Ma Chung Research Center for Photosynthetic Pigments,

Ma Chung University,

Malang, Indonesia

H. Scheer

Department of Biology, Ludwig-Maximilian-University,

Großhaderner Str. 2,

80638 Munich, Germany

\section{Introduction}

Plasmon excitations in metallic nanoparticles provide an excellent way to control the optical properties of matter at the nanoscale [1]. Hybrid nanostructures comprising metallic nanoparticles have been studied in many fields such as optoelectronics [2], biosensors [3], cancer therapy [4], or photovoltaics [5]. One of the prerequisites for controlling the properties at the nanoscale using plasmonic excitations is the ability to fabricate an on-demand hybrid plasmonic nanostructure with well-defined characteristics. As the strength of the interaction between plasmons and other nanostructures depends upon the spectral properties of both components as well as the geometry of a hybrid nanostructure, it is required to research the fundamental interactions between plasmons localized in metallic nanoparticles with optically active molecules. Placing a fluorescent molecule in the vicinity of a metallic nanoparticle may either quench or enhance the fluorescence [6] depending on the interplay between the plasmon-induced increase of both fluorescence quantum yield and the absorption rate and non-radiative energy transfer from the molecule to the nanoparticle. Typically, the latter process becomes dominant for distances below $5 \mathrm{~nm}$ : the emission is efficiently quenched. On the other hand, the largest enhancements of the fluorescence have been observed for distances between 10 and $30 \mathrm{~nm}$ between the metallic nanoparticle and the fluorescent molecule [7].

While spectroscopy studies of hybrid plasmonic nanostructures comprising only one type of fluorescentmolecule are currently in an advanced stage, extending both the techniques and understanding toward more complicated systems such as multi-pigment biomolecules still remains a challenge. A major difficulty concerns the interactions between the pigments in multi-chromophoric systems, for instance fluorescence reso- 
nant energy transfer, and the preparation of biological systems in a state that is suitable for spectroscopic studies of plasmoninduced effects.

Recently, several reports have focused on the effect of local electromagnetic field generated in metallic nanoparticles on the optical properties of chlorophyll-containing biological light-harvesting complexes [8-12]. When peridinin-chlorophyll-protein (PCP) and Photosystem I (PSI) were coupled to a highly inhomogeneous ensemble of metallic nanoparticles, the fluorescence $[8,9]$ and/or absorption [10] can be increased. The influence of plasmonic interaction on the protein function has also been studied for the latter PSI lightharvesting system [11] and the fluorescence enhancement for allophycocyanin deposited on silver island film has been observed [12].

In this work, we attempt to optimize the geometry of a plasmonic hybrid nanostructure such that the electromagnetic field generated in metallic nanoparticles leads exclusively to the enhancement of absorption of the lightharvesting complex. The structure comprises a layer of semicontinuous silver film with a relatively narrow size distribution that is separated from the light-harvesting complexes by a 25 -nm-thick $\mathrm{SiO}_{2}$ layer. Large energy separation between the absorption and fluorescence of the light-harvesting complex, PCP, combined with a properly designed metallic nanostructure, with well-defined plasmon resonance, offers the unique opportunity to separate the influence of plasmonic excitation on the excitation rate from that on the emission. The fluorescence excitation spectra show a very strong absorption band in the spectral range corresponding to the plasmon resonance of the silver islands, which could be modeled by the electromagnetic field enhancement generated in such a hybrid nanostructure. On the other hand, fluorescence decay times change only slightly upon placing the light-harvesting complexes on the silver island film. We conclude that the design of our structure provides an efficient way to plasmonically enhance the absorption of light-harvesting complexes.

\section{Samples and Experiment}

Light-harvesting complexes, or antennas, enhance and broaden the absorption of photosystems for the efficient use of sunlight in photosynthesis. PCP is one of many such complexes. It is a water-soluble biomolecule employed by Dinoflagellates as an antenna external to the membrane [13]. The structure of the PCP complex has been determined with 1.3-A resolution using X-ray crystallography. The native form of PCP consists of two chlorophyll $a$ (Chl) and eight peridinin (Per) molecules [13]. All pigments are embedded in a protein matrix. As the ratio of Per to $\mathrm{Chl}$ is $>>1$, in contrast to most of other light-harvesting complexes, PCP utilizes the carot- enoid, Per, and not the Chls as its main light-harvesting pigment. The PCP complex can be reconstituted with other Chl dervatives which, for example, exhibit different optical properties $[14,15]$. One example is [3-acetyl]-chlorophyll $a$ (acChl). Chemically, it differs from Chl only by the C-3 substituent, but the absorption and fluorescence spectra of acChl-PCP, the PCP complex reconstituted with acChl, is red-shifted as compared with the PCP complex containing Chl. At the same time, the Per absorption in the blue-green spectral range is affected very slightly [14]. The advantage of using a red-shifted pigment stems from the better spectral separation between the emission of the pigment and the Per absorption to which the plasmon resonance frequency of the metallic nanoparticles used to fabricate the plasmonic hybrid nanostructure is matched.

The peridinin-chlorophyll-protein complexes used in our experiments were prepared as described [16]. In order to obtain PCP complexes containing Chl, the N-terminal apoprotein was reconstituted with an appropriate mixture of Per and, as desired, Chl or acChl. The reconstituted monomeric PCP was equilibrated with TRIS buffer and kept frozen until used. The absorption (and fluorescence excitation) spectrum of the acChl-PCP has an intense, broad band between 400 and $550 \mathrm{~nm}$ that is due to Per and two Chl bands at $440 \mathrm{~nm}$ (Soret) and $690 \mathrm{~nm}\left(\mathrm{Q}_{\mathrm{Y}}\right)$, the emission spectrum peaks at $690 \mathrm{~nm}$ (Fig. 1a) [16].

The semicontinuous silver films were deposited onto a glass coverslip utilizing the e-beam evaporation technique [17]. The thickness of the silver layer was nominally $7 \mathrm{~nm}$. In order to protect the metallic layer and to diminish the impact of the non-radiative energy transfer from the PCP complexes to the silver islands, a 25-nm-thick layer of silica was deposited using e-beam evaporation $[17,18]$. The e-beam evaporation method allows for a much more homogeneous distribution of the size of silver islands compared, in particular, with the methods used previously $[8,9,19]$. The absorption spectrum of such a silver island film (Fig. 1b) shows the plasmon resonance around $450 \mathrm{~nm}$ that matches very well the Per absorption of the PCP complex (Fig. 1a). Previous SEM studies of the identically prepared semicontinuous silver film [17] have demonstrated the relatively good homogeneity of the plasmonic structure.

A $2-\mu 1$ drop of the acChl-PCP solution was deposited directly onto the metallic substrate and then slowly dried at room temperature in darkness to inhibit the degradation of the light-harvesting complexes. In comparison with previous experiments $[8,9]$, where a polymer layer was used, the present approach assures that most of the PCP complexes are at the $\mathrm{SiO}_{2}$ surface; thus, their distance between the PCP complexes and metallic nanoparticles is very uniform and defined by the thickness of the $\mathrm{SiO}_{2}$ layer. For the reference, a similar sample was prepared with PCP complexes deposited directly on a clean glass coverslip. 

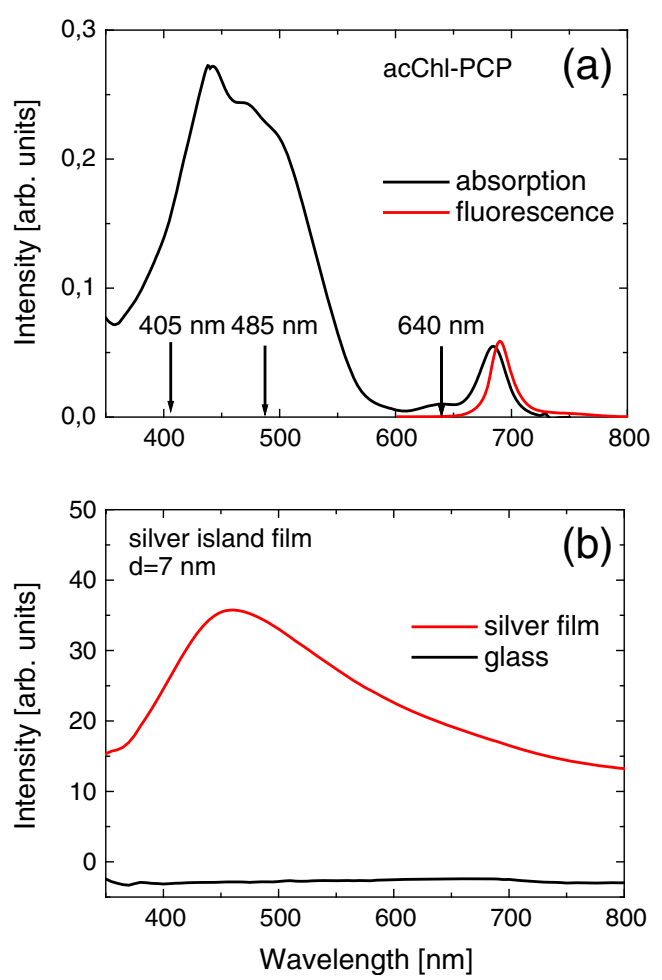

Fig. 1 a Absorption (black) and fluorescence spectra (red, $\lambda_{\mathrm{exc}}=$ $532 \mathrm{~nm}$ ) of acChl-PCP complexes. Arrows mark the wavelengths used for time-resolved experiment. b Absorption spectrum of the semicontinuous silver film (red) compared to the absorption spectrum of the glass substrate (black)

The fluorescence and fluorescence excitation spectra of both structures were measured using the FluoroLog 3 spectrofluorimeter (Jobin Yvon) equipped with a specially designed mount suitable for holding planar samples. A xenon lamp source with a double-grating monochromator was used for excitation, and the signal was detected with a thermoelectrically cooled photomultiplier tube characterized by a dark current of $<100 \mathrm{cps}$. The experiments were carried out in a backscattering geometry. The decay times of fluorescence were measured using time-correlated singlephoton counting. The experimental setup was based on the SPC-150 card (Becker \& Hickl). For the excitation, 3-ps lasers were used with the wavelengths of 405,485 , and $640 \mathrm{~nm}$, each operating at an $80 \mathrm{-MHz}$ repetition rate; the average excitation power was about $2 \mathrm{~mW}$. The detection was carried out using an idQ-100-50-ULN silicon avalanche photodiode detector (idQuantique). The time resolution of the setup was around $250 \mathrm{ps}$. The fluorescence of the acChl-PCP complexes was extracted using a band-pass filter HQ690/20 (Chroma).

The three excitation wavelengths (marked by arrows in Fig. 1a) provide a means to study the behavior of $\mathrm{Chl}$ fluorescence for qualitatively different conditions of plasmon coupling. Excitation at $405 \mathrm{~nm}$ corresponds mainly to the Soret band of acChl absorption, excitation at $485 \mathrm{~nm}$ corresponds to the Per absorption, and excitation at $640 \mathrm{~nm}$ corresponds to $Q_{\mathrm{Y}}$ absorption of the acChls. In the latter, Per molecules are not excited. Moreover, whereas the two first lasers efficiently excite plasmon resonance in the silver island film, the wavelength of $640 \mathrm{~nm}$ overlaps very weakly with the plasmon spectrum of the silver nanoparticles.

\section{Results and Discussion}

The effect of plasmon excitations in metallic nanoparticles on the optical properties of nearby emitters is commonly monitored by its fluorescence intensity. In the case of plasmon-induced enhancement, the fluorescence intensity of such a hybrid structure is increased [6]. When, on the other hand, non-radiative energy transfer from the emitter to the metallic nanoparticles plays the dominant role, the emission is efficiently quenched. This gives only incomplete information, however, because the fluorescence depends upon the spectral properties of the emitter and their relation to the plasmon resonances in the metallic nanoparticles. Additional information about plasmonic interactions can be extracted by combining fluorescence excitation spectroscopy [20] and time-resolved fluorescence spectroscopy; these two experimental techniques provide a way to separate the plasmon-induced increase of the radiative rate from an induced increase of the absorption. The acChl-PCP complex is well suited for such a study because of its large Stokes shift when the carotenoids are excited (Fig. 1).

In Fig. 2 we compare the fluorescence excitation spectra of acChl -PCP deposited on the semicontinuous silver film and on the glass substrate, also shown is the emission spectrum $\left(\lambda_{\mathrm{exc}}=532 \mathrm{~nm}\right)$. The excitation spectrum for the reference sample looks very similar to previously published data [14]; it features a strong absorption due to Per in the spectral range from 400 to $550 \mathrm{~nm}$ and corresponds roughly to the absorption spectrum (Fig. 1a). This indicates that the sample preparation process affects neither the protein nor the pigments. In contrast, the maximum is blue-shifted by $\sim 40 \mathrm{~nm}$ for the PCP complexes deposited on the silver island film and separated from the metallic nanostructures by a 25 -nm-thick $\mathrm{SiO}_{2}$ layer. After subtracting both curves (Fig. 2b), we find that the difference has a shape of the band with a maximum at $407 \mathrm{~nm}$ and linewidth of about $35 \mathrm{~nm}$. We conclude that the observed enhancement is due to plasmon excitations in the metallic layer: this observation is a central result of this work.

The fluorescence excitation spectra displayed in Fig. 2a have not been in any way adjusted or normalized. They are very similar to each other for wavelengths longer than $475 \mathrm{~nm}$, in particular in the absorption range of low-energy 



Fig. 2 a Fluorescence excitation spectra measured for the acChl-PCP complexes deposited on the semicontinuous silver film substrate (red) compared with that of the reference sample (green). The detection wavelength was $690 \mathrm{~nm}$. b Difference between the two spectra displayed in (a)

Per molecules. This suggests that the number of PCP complexes probed in both experiments is almost identical, which makes the estimation of the enhancement factor remarkably straightforward. Importantly, the fluorescence spectrum of the acChl-PCP complexes deposited on the semicontinuous silver film is identical, for all excitation wavelengths, to that of the reference structure, which supports our previous observation that the preparation of the hybrid nanostructure has no measureable effect on the protein or pigment structure.

The time-resolved fluorescence of the two samples is compared in Fig. 3 for excitation wavelengths of (a) $405 \mathrm{~nm}$, (b) $485 \mathrm{~nm}$, and (c) $640 \mathrm{~nm}$. In this way, we probe the fluorescence dynamics when plasmons in the semicontinuous silver film are weakly excited (640-nm excitation) and when they are efficiently excited (405 and $485 \mathrm{~nm}$ ). The decay kinetics of the control sample on glass are $3.25 \pm$ $0.28 \mathrm{~ns}$ irrespective of the excitation wavelength (Table 1). A similar value is also obtained for the sample on the silver islands with an excitation at $640 \mathrm{~nm}$. The lifetime is, in contrast, decreased to $2.28 \pm 0.2 \mathrm{~ns}$ when plasmons in the silver island film are excited. While measurable, this change is relatively small compared with previous results on fluorescent dyes [21] and light-harvesting complexes [8,9],
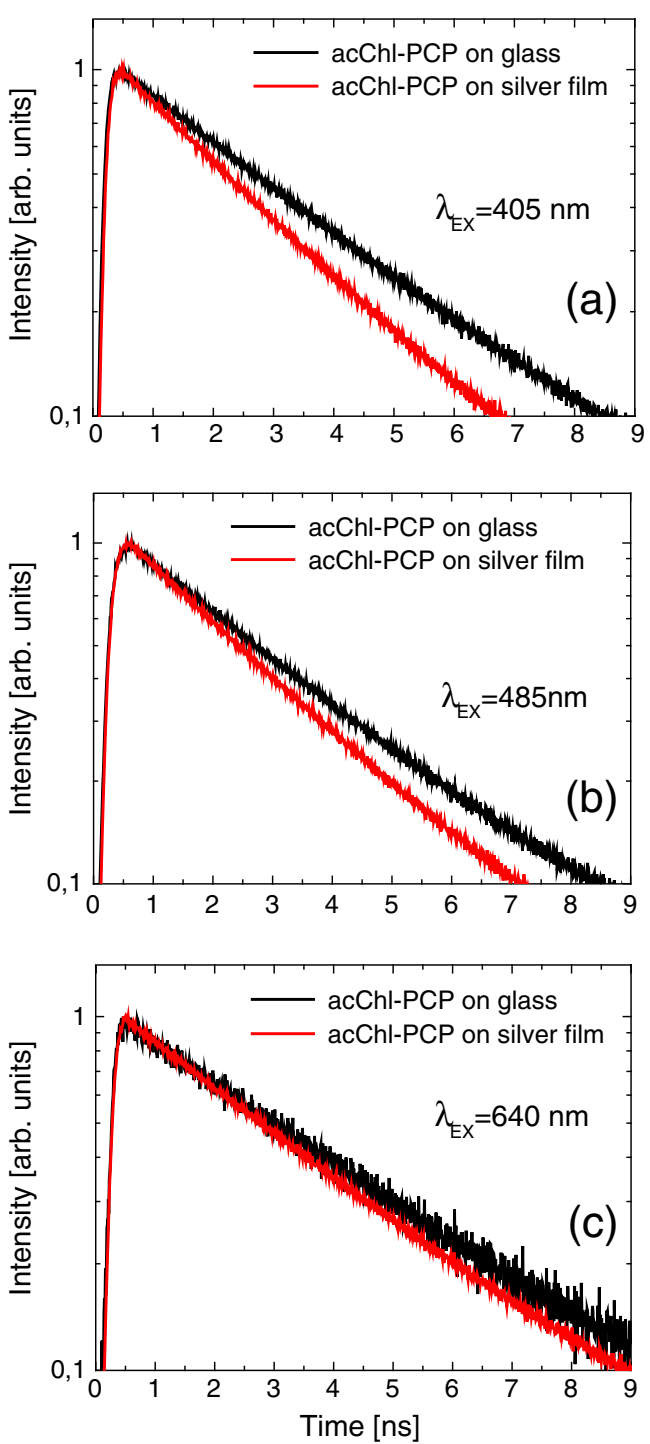

Fig. 3 Fluorescence kinetics for the acChl-PCP complexes deposited on the metallic substrate (red) compared with the ones obtained for the reference sample on glass (black). The excitation wavelengths were $405 \mathrm{~nm}(\mathbf{a}), 485 \mathrm{~nm}(\mathbf{b})$, and $640 \mathrm{~nm}(\mathbf{c})$

where order-of-magnitude changes have been measured. The small change of the fluorescence lifetimes in the case of the PCP complexes coupled to the silver island film supports our conclusion that the enhancement measured in the fluorescence excitation (Fig. 1) is predominantly due to the enhancement of the excitation rate in the light-harvesting complex. At the same time, the dependence of the fluorescence decay on the excitation wavelength for the PCP complexes deposited onto the metallic islands suggests that the non-plasmon-related influence of the metallic film upon the studied biomolecule is negligible.

In order to model the observed enhancement of both the absorption and emission of the PCP complexes deposited onto the silver island film, we used the boundary element method [22]. This approach allows for calculating the total 
Table 1 Fluorescence decay times measured for acChl-PCP complexes deposited on glass and semicontinuous silver substrates

\begin{tabular}{lccc}
\hline Excitation wavelength (nm) & 405 & 485 & 640 \\
acChl-PCP on glass substrate (ns) & 3.18 & 3.02 & 3.56 \\
acChl-PCP on SIF substrate (ns) & 2.28 & 2.33 & 3.1 \\
Change (\%) & 28 & 23 & 13 \\
\hline
\end{tabular}

The percentage change for the three excitation wavelengths used in the experiment is given. The error for estimating the values of fluorescence decay times is approximately $0.25 \mathrm{~ns}$

electric field intensity, $E_{\mathrm{tot}}$, on the top surface of the silicon dioxide spacer for a predefined geometry of the metallic nanoparticle layer. This total electric field is a sum of an electric component of incident laser light, $E_{\text {inc }}$, and the field scattered by the silver nanostructures, $E_{\text {scatt }}$. We define the enhancement factor $\kappa$ as [23, 24]:

$\kappa \sim \frac{<\left|E_{\text {tot }}\right|^{2}>}{\left|E_{\text {inc }}\right|^{2}}$

where $<\left|E_{\text {tot }}\right|^{2}>$ corresponds to the average total electric field on the top surface of the silica spacer. We introduce the average value of the electric field for the purpose of estimating the electromagnetic enhancement not only for isotropic (spherical) nanoparticles but also for anisotropic geometries. Indeed, we carried out the analysis for three qualitatively different arrangements of metallic nanoparticles: uncoupled spherical islands, coupled dimers of spherical nanoparticles, and elongated nanoparticles, that is nanorods.

While for a single metallic nanosphere the resonance frequency is independent of the direction, the other two geometries have a reduced symmetry. This leads to two characteristic frequencies; therefore, the calculated enhancement factor must be averaged over two polarizations of incident electric field (along the symmetry axes of the respective metallic nanoparticle arrangement). For all cases, the sizes of silver nanostructures were chosen to approximately match the actual size of the silver islands as measured using the SEM technique [17]. The diameter (width) of spherical (elongated) nanoparticles was in the range of 5$20 \mathrm{~nm}$. The results for all three cases are displayed in Fig. 4, where spectral dependence of $\kappa$ is shown.

In the case of spherical nanoparticles (Fig. 4a), we obtained a single electric dipolar resonance at $\lambda \approx 400 \mathrm{~nm}$, which matches well with the spectral dependence of the fluorescence enhancement observed experimentally for the hybrid nanostructure comprising light-harvesting complexes. The spectral position of this resonance is virtually independent of the size of the nanoparticle in the range studied.

For dimers of spherical nanoparticles (Fig. 4b), we observe two effects: firstly, the energies of calculated resonances strongly depend upon the polarization of the
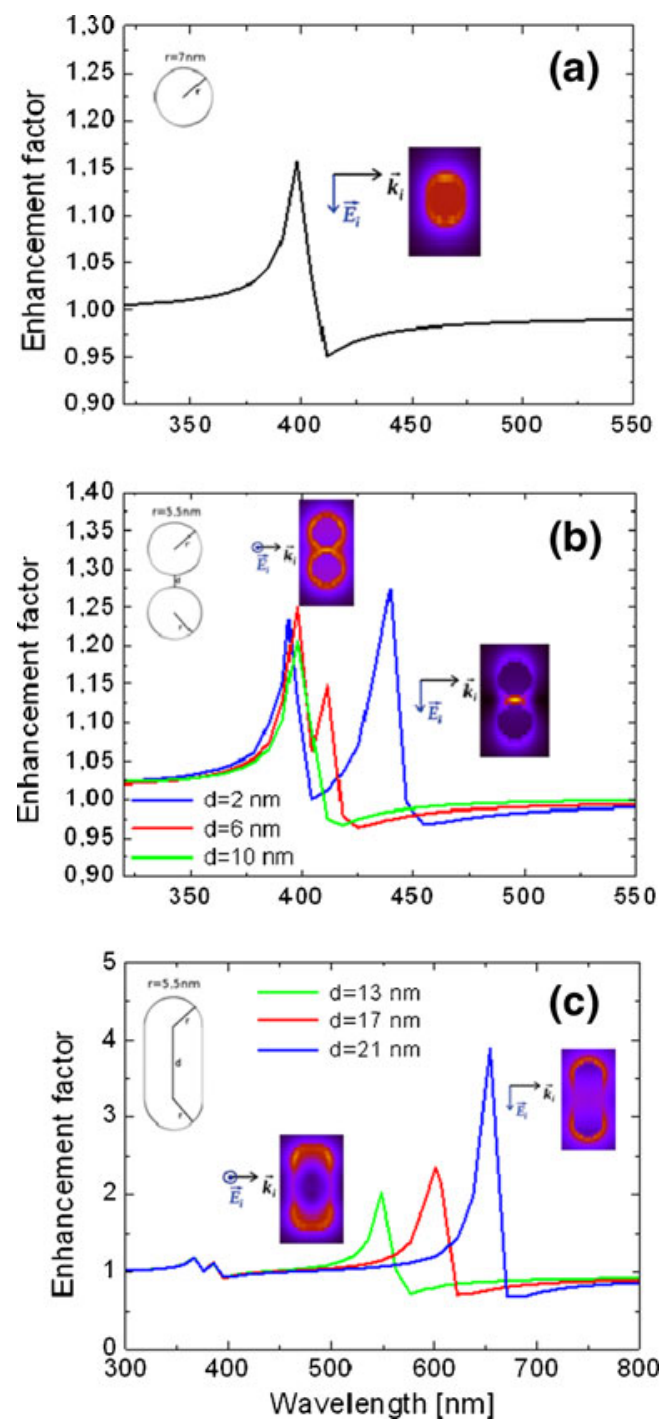

Fig. 4 Enhancement factor $\kappa$ of electric field intensity calculated on the surface of a 25 -nm-thick silica spacer as a function of incident light wavelength for three morphologies of plasmonic structures: isolated spherical nanoparticle (a), coupled spherical nanoparticles (b), and metallic nanorods (c). Calculations for coupled metallic nanoparticles and nanorods were carried out for three cases of separation and length, as indicated. The insets show the distribution of the electric field in the cross section of the structures calculated for the resonance wavelengths

incident light, and secondly, the splitting between the two resonances increases with a larger separation between the two spherical nanoparticles $[25,26]$. The first effect was taken into account when estimating the total electromagnetic field enhancement by averaging over the two directions. On the other hand, by gradually increasing the distance between the two nanoparticles forming a dimer from 2 to $10 \mathrm{~nm}$, we induce blue shifts of the resonance frequencies of up to $50 \mathrm{~nm}$, which reflects the weaker coupling of the nanoparticles at longer distances. 
The results obtained for metallic nanorods (Fig. 4c) are in a way more extreme than those of coupled spherical nanoparticles. We assumed a constant radius of $5.5 \mathrm{~nm}$ of the endcaps that were modeled as hemispheres, while the length of the nanorod changed from 13 to $21 \mathrm{~nm}$. In this case, we observe a strong polarization selectivity of the two plasmon resonances, with the lower-energy one yielding much larger enhancement factors and shifting as far as to $700 \mathrm{~nm}$ for the longest nanorods. The energy of the strong, red-shifted resonance depends linearly upon the length of the nanorod. On the other hand, the weaker resonance appears around $400 \mathrm{~nm}$ and is almost independent of the nanorod length [27].

We now consider the implications of the effects observed for the three geometries of plasmonic nanostructures for understanding the impact of plasmon resonances in the silver island film on the optical properties of the PCP complexes. While the nanorods feature strong resonances in the spectral region comparable to the fluorescence emission range of the PCP complexes, coupling to such structures would result in a strong enhancement of the radiative rate, hence substantial shortening of the fluorescence decay time. In contrast, the enhancement in the 400- to 500-nm spectral range is minute for metallic nanorods. Therefore, we conclude that such geometry is highly unlikely in the studied structure. On the other hand, a moderate, approximately twofold enhancement observed experimentally can be well explained by assuming the presence of isolated and coupled spherical nanoparticles in the silver film structure. Indeed, they exhibit plasmon resonances in the range of $400-450 \mathrm{~nm}$. Furthermore, since the strength of the interaction between metallic nanoparticles is not homogeneous, some distribution of the spectral shifts toward longer wavelengths is expected. These can cause broadening of the enhancement curve observed experimentally for the studied hybrid nanostructure.

\section{Conclusions}

We studied the electromagnetic field enhancement in a hybrid nanostructure composed of light-harvesting complexes deposited on a semicontinuous silver film separated by a $25-\mathrm{nm}$-thick silica spacer. The increase of the acChlPCP absorption in the spectral range comparable to the plasmon resonances in metallic nanoparticles indicates strong enhancement of absorption due to plasmon interactions. The combination of fluorescence excitation and time-resolved fluorescence spectroscopy allows for separating contributions of absorption and fluorescence rate enhancements. A comparison between the experimental results and calculations for various morphologies indicates that the semicontinuous silver film consists predominantly of isolated islands and/or coupled ones.
Acknowledgments Research in Poland has been supported by the WELCOME project "Hybrid Nanostructures as a Stepping Stone towards Efficient Artificial Photosynthesis" funded by the Foundation for Polish Science and EUROCORES project "BOLDCATS" funded by the European Science Foundation. THPB acknowledges support from the US Department of Energy (DE-SC001035), which established the Photosynthetic Antenna Research Center (PARC).

Open Access This article is distributed under the terms of the Creative Commons Attribution Noncommercial License which permits any noncommercial use, distribution, and reproduction in any medium, provided the original author(s) and source are credited.

\section{References}

1. Maier SA (2007) Plasmonics: fundamentals and applications, 1st edn. Springer, New York

2. Novotny L, van Hulst N (2011) Antennas for light. Nature Phot 5:83-90

3. Lee J, Hernandez P, Lee J, Govorov AO, Kotov NA (2007) Exciton-plasmon interactions in molecular spring assemblies of nanowires and wavelength-based protein detection. Nature Mat 6:291-295

4. Huang X, El-Sayed IH, Qian W, El-Sayed MA (2006) Cancer cell imaging and photothermal therapy in the near-infrared region by using gold nanorods. J Am Chem Soc 128:2115-2120

5. Atwater HA, Polman A (2010) Plasmonics for improved photovoltaic devices. Nature Mat 9:205-213

6. Anger P, Bharadwaj P, Novotny L (2006) Enhancement and quenching of single-molecule fluorescence. Phys Rev Lett 96:113002

7. Lakowicz JR (2006) Plasmonics in biology and plasmoncontrolled fluorescence. Plasmonics 1:5-33

8. Mackowski S, Wörmke S, Maier AJ, Brotosudarmo THP, Harutyunyan H, Hartschuh A, Govorov AO, Scheer H, Bräuchle C (2008) Metal-enhanced fluorescence of chlorophylls in single light-harvesting complexes. Nano Lett 8:558-564

9. Mackowski S (2010) Hybrid nanostructures for efficient light harvesting. J Phys: Condens Matter 22:193102/1-17

10. Carmeli I, Liberman I, Kraverski L, Fan Z, Govorov AO, Markovich G, Richter S (2010) Broad band enhancement of light absorption in photosystem I by metal nanoparticle antennas. Nano Lett 10:2069-2074

11. Nieder JB, Bittl R, Brecht M (2010) Fluorescence studies into the effect of plasmonic interactions on protein function. Angew Chem Int Ed 49:10217-10220

12. Chowdhury MH, Ray K, Aslan K, Lakowicz JR, Geddes CD (2007) Metal-enhanced fluorescence of phycobiliproteins from heterogeneous plasmonic nanostructures. J Phys Chem C 111:18856-18863

13. Hofmann E, Wrench PM, Sharples FP, Hiller RG, Welte W, Diederichs K (1996) Structural basis of light harvesting by carotenoids: peridinin-chlorophyll-protein from Amphidinium carterae. Science 272:1788-1791

14. Polívka T, Pascher T, Sundström V, Hiller RG (2005) Tuning energy transfer in the peridinin-chlorophyll complex by reconstitution with different chlorophylls. Photosynth Res 86:217-227

15. Brotosudarmo THP, Hofmann E, Hiller RG, Wörmke S, Mackowski S, Zumbusch A, Bräuchle C, Scheer H (2006) Peridininchlorophyll-protein reconstituted with chlorophyll mixtures: preparation, bulk and single molecule spectroscopy. FEBS Lett 580:5257-5262

16. Brotosudarmo THP, Mackowski S, Hofmann E, Hiller RG, Bräuchle C, Scheer H (2008) Relative binding affinities of 
chlorophylls in peridinin-chlorophyll-protein reconstituted with heterochlorophyllous mixtures. Photosynth Res 95:247-252

17. Chettiar UK, Nyga P, Thoreson MD, Kildishev AV, Drachev VP, Shalaev VM (2010) FDTD modeling of realistic semicontinuous metal films. Appl Phys B 100:159-168

18. Thoreson MD, Fang J, Kildishev AV, Prokopeva LJ, Nyga P, Chettiar UK, Shalaev VM, Drachev VP (2011) Fabrication and realistic modeling of three-dimensional metal-dielectric composites. J Nanophoton 5:051513

19. Lakowicz JR, Shen Y, D'Auria S, Malicka J, Fang J, Gryczynski Z, Gryczynski I (2002) Radiative decay engineering: 2. Effects of silver island films on fluorescence intensity, lifetimes, and resonance energy transfer. Anal Biochem 301:261-277

20. Lee J, Javed T, Skeini T, Govorov AO, Bryant GW, Kotov NA (2006) sBioconjugated Ag nanoparticles and CdTe nanowires: metamaterials with field-enhanced light absorption. Angew Chem Int Ed 45:4819-4823

21. Dulkeith E, Morteani AC, Niedereichholz T, Klar TA, Feldmann J, Levi SA, van Veggel FCJM, Reinhoudt DN, Möller M, Gittins DI (2002) Fluorescence quenching of dye molecules near gold nanoparticles: radiative and nonradiative effects. Phys Rev Lett 89:203002

22. García de Abajo FJ, Howie A (1998) Relativistic electron energy loss and electron-induced photon emission in inhomogeneous dielectrics. Phys Rev Lett 80:5180-5183

23. Govorov AO, Bryant GW, Zhang W, Skeini T, Lee J, Kotov NA, Slocik JM, Naik RR (2006) Exciton-plasmon interaction and hybrid excitons in semiconductor-metal nanoparticle assemblies. Nano Lett 6:984-994

24. Gersten J, Nitzan A (1984) Spectroscopic properties of molecules interacting with small dielectric particles. J Chem Phys 75:1139-1152

25. Bek A, Jansen R, Ringler M, Mayilo S, Klar T, Feldmann J (2008) Fluorescence enhancement in hot spots of AFM-designed gold nanoparticle sandwiches. Nano Lett 8:485-490

26. Gunnarsson L, Rindzevicius T, Prikulis J, Kasemo B, Kall M, Zou S, Schatz GC (2005) Confined plasmons in nanofabricated single silver particle pairs: experimental observations of strong interparticle interactions. J Phys Chem B 109:1079-1087

27. Bryant GW, García de Abajo FJ, Aizpurua J (2008) Mapping the plasmon resonances of metallic nanoantennas. Nano Lett 8:631-636 